\title{
Bone Biomineral Properties Vary Across Human Osteonal Bone.
}

\author{
Nina K. Wittig ${ }^{1}$, Jonas Palle ${ }^{1}$, Maja Østergaard ${ }^{1}$, Simon Frølich ${ }^{1}$, Mie E. Birkbak ${ }^{1}$, Kathryn M. Spiers ${ }^{2}$, \\ Jan Garrevoet ${ }^{2}$, Henrik Birkedal ${ }^{1 *}$ \\ ${ }^{1}$ Department of Chemistry and iNANO, Aarhus University, Gustav Wieds Vej 14, 8000 Aarhus C, Denmark. \\ ${ }^{2}$ DESY Photon Science, Notkestr. 85, D-22607 Hamburg, Germany \\ *hbirkedal@chem.au.dk.
}

\begin{abstract}
The biomineralization of bone remains a puzzle. During Haversian remodeling in the dense human cortical bone, osteoclasts excavate a tunnel that is then filled in by osteoblasts with layers of bone of varying fibril orientations, resulting in a lamellar motif. Such bone represents an excellent possibility to increase our understanding of bone as a material as well as bone biomineralization by studying spatio/temporal variations in the biomineral across an osteon. To this end, fluorescence computed tomography and diffraction scattering computed tomography with sub-micrometer resolution is applied to obtain position resolved fluorescence spectra and diffraction patterns in a 3D volume. The microstructural properties of the apatite biomineral are not homogeneous but depends critically on the time point at which it was laid down. This indicates that the nature of bone biomineral is highly dependent on the microenvironment during bone formation and remodeling.
\end{abstract}

KEYWORDS. biomineralization, hierarchical materials, diffraction scattering computed tomography, fluorescence computed tomography, bone

Bone continues to puzzle researchers and remains far from understood due to its hierarchical, but complex architecture and the dynamic nature of bone imposed by the fact that it is a living

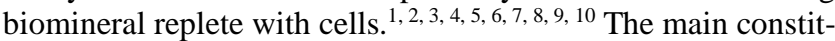
uents of bone are collagen fibrils, nano-crystalline and highly substituted hydroxyapatite and water. ${ }^{11}$ Additionally bone also contains a multitude of non-collagenous proteins and other organic molecules. The biogenic hydroxyapatite crystallizes in the hexagonal space group $P 6_{3} / \mathrm{m}^{12,}{ }^{13}$ In humans and certain other animals, the dense cortical bone is remodeled by Haversian remodeling to form osteons ${ }^{14}$ in a concerted process involving bone-degrading osteoclasts and bone-forming osteoblasts. ${ }^{15}$ For unknown reasons, Haversian remodeling only occurs in some animals and, for example, not in rodents. ${ }^{5,16,17}$ During Haversian remodeling, osteoclasts carve out a tunnel, which is consecutively filled in by osteoblasts with layers of bone with a winding pattern of mineralized collagen fibrils ${ }^{15,18,19}$ forming one after another from the outside inwards. This results in a pseudo-periodic modulation in mineral density and local stiffness on the $1-10 \mu \mathrm{m}$ scale. ${ }^{20}$ During bone formation, some osteoblasts are buried in the bone matrix to form the osteocyte lacuno-canalicular network. ${ }^{21,22}$ The importance of Haversian remodeling is unclear. Osteonal bone is less stiff than the interstitial bone laid down initially and several theories have been proposed to explain why some animal species form osteons while others do not. ${ }^{5,23,24}$ Osteons are predominantly oriented parallel to the bone long axis contributing to the highly anisotropic macro-mechanical properties of the whole bone. ${ }^{25,26,27,28}$
It remains unclear how cellular control over biomineral formation occurs, for example, how does the physico-chemical milieu at the time of biomineralization influence the forming biomineral? Since osteoblasts form consecutive layers of material from the outside in during osteon formation, we hypothesized that the spatio/temporal variations in bone biomineralization can be followed by mapping bone biomineral structure across an osteon using X-ray diffraction and fluorescence. To this end, a resolution better than one lamella, about $1 \mu \mathrm{m}$, in three dimensions is needed. For 2D mapping, such experiments would require equally thin specimens that are difficult to produce and give extremely low diffraction signals. Therefore, we employed fluorescence computed tomography (XRF-CT) $)^{29,30}$ and diffraction scattering computed tomography (DSCT) ${ }^{31}, 32,33,34,35,36$ with a sub-micron X-ray beam to enable measurement of X-ray fluorescence spectra and powder diffraction patterns, respectively, within a human osteon in 3D. This revealed that bone biomineral crystallographic properties such as lattice parameters, crystallite size, and local disorder depend on where/when in the osteon mineralization process the biomineral was formed.

\section{RESULTS AND DISCUSSION}

We measured XRF-CT and DSCT in two separate experiments with a $400 \mathrm{~nm}$ diameter X-ray beam at $16.5 \mathrm{keV}$ (see Methods section for details). Fluorescence was measured on a thin ( 30 $\mu \mathrm{m})$ approximately octagonal rod cut perpendicular to the osteon central canal, while the diffraction data were collected on a 130 $\mu$ m diameter pseudo-octagonal rod parallel to the osteon axis, see Figure 1A. The obtained fluorescence/diffraction sig- 
nals were reconstructed tomographically to obtain 3D distributions of fluorescence intensities and powder diffractograms, respectively. ${ }^{31,33,34,35,37,38,39}$ Both experiments were conducted with $(400 \mathrm{~nm})^{3}$ voxel size. The fluorescence experiment covered a $46 \times 46 \times 126 \mu^{3}$ volume ( $>4.2$ million voxels), while the diffraction experiment covered 26 consecutive layers resulting in a total measured volume of $150 \times 150 \times 10.4 \mu^{3}$ (>3.6 million voxels).

Figures 1B-E show the main XRF-CT results. The reconstructed Ca fluorescence intensity (Figure 1B) reveals the presence of osteocyte void spaces (lacunae) and cellular connections (canaliculi), which are rendered in 3D in Figure 1D. The cellular connections are only a few hundred nm wide, ${ }^{21,40,41}$ indicating that indeed sub-micrometer resolution was achieved. As quantified in Figure 1E, the Ca concentration was higher close to the osteon central canal than in the major part of the osteon. It was also higher in surrounding bone (interstitial bone) with a clear transition at the cement line outlining the osteon that was even sharper when mapping fluorescence as a function of distance from the cement line (Figure S1, Supporting Information). This difference reflects that the osteon does not have concentric circular symmetry (see below). The higher levels of $\mathrm{Ca}$ in interstitial than in osteonal bone, corresponding to a higher degree of mineralization is well-established. ${ }^{42}$ The increase in Ca levels in interstitial bone is accompanied by an even larger relative increase in Sr levels (Figure 1B, E, S1A-B), which is in correspondence with 2D X-ray fluorescence studies showing that the concentration of $\mathrm{Sr}$ increases with increasing mineralization. ${ }^{43}$ That study also showed that $\mathrm{Sr}$ is heterogeneously accumulated among different structural units in bone. ${ }^{43}$ The small increase in the Sr levels inside the particular osteon investigated in the present study approximately halfway between the central canal and the cement line suggests a heterogeneous uptake of $\mathrm{Sr}$ even within structural units. When bone is treated with Sr-containing drugs, $\mathrm{Sr}$ is incorporated into bone nanocrystals. ${ }^{44,45,46}$ The local Sr levels are thus likely a testament of the chemical composition of the mineralizing medium during bone formation.

The diffraction data were analyzed by Rietveld refinement of each of the over 3.6 million reconstructed diffraction patterns (Supporting Information), ${ }^{47,48,49}$ which is almost two times more than the previous largest DSCT Rietveld scheme ${ }^{50}$ but with a $>60$ times smaller beam. A simplified Rietveld model incorporating the amount of crystalline material (scale factor), a varying background signal, apatite lattice constants, peak full width half maximum (FWHM) parameters corresponding to needle shaped nanocrystals, and an average atomic displacement parameter, $U_{\text {iso }}$, that describes the combined effect of average atomic thermal motion and disorder in the apatite lattice. Examples of fitted data are shown in Figure S2.

Note that the diffraction experiment provides information about the average structure in each voxel, and the parameters extracted such as the lattice parameters thus also represent an average. Variations in lattice parameters across the reconstructed 3D volume are expected to mostly arise from differences in chemical substitutions, but it is exceedingly difficult to predict how in the heavily substituted bone apatite, ${ }^{13,51,52}$ and, but to a smaller extent, due to macro-strain effects from tightly bound or occluded biomolecules. ${ }^{53,54}$ The width of diffraction peaks is determined by crystallite size and micro-strain fluctuation effects as well as instrumental effects. ${ }^{55}$ The latter do not depend on position so any changes seen across the sample must stem from the finite size of the crystallites, with increasing broadening with decreasing crystallite size, or micro-strain fluctuation effects. Micro-strain fluctuations arises from variations in lattice parameters within a coherently scattering crystallite opposed to macro-strain that arises from variations in the average lattice parameters between crystallites. The former thus causes peak broadening, while the latter results in a peak shift. Due to the hexagonal symmetry of biogenic HAP $(a=b)^{12,13}$ it

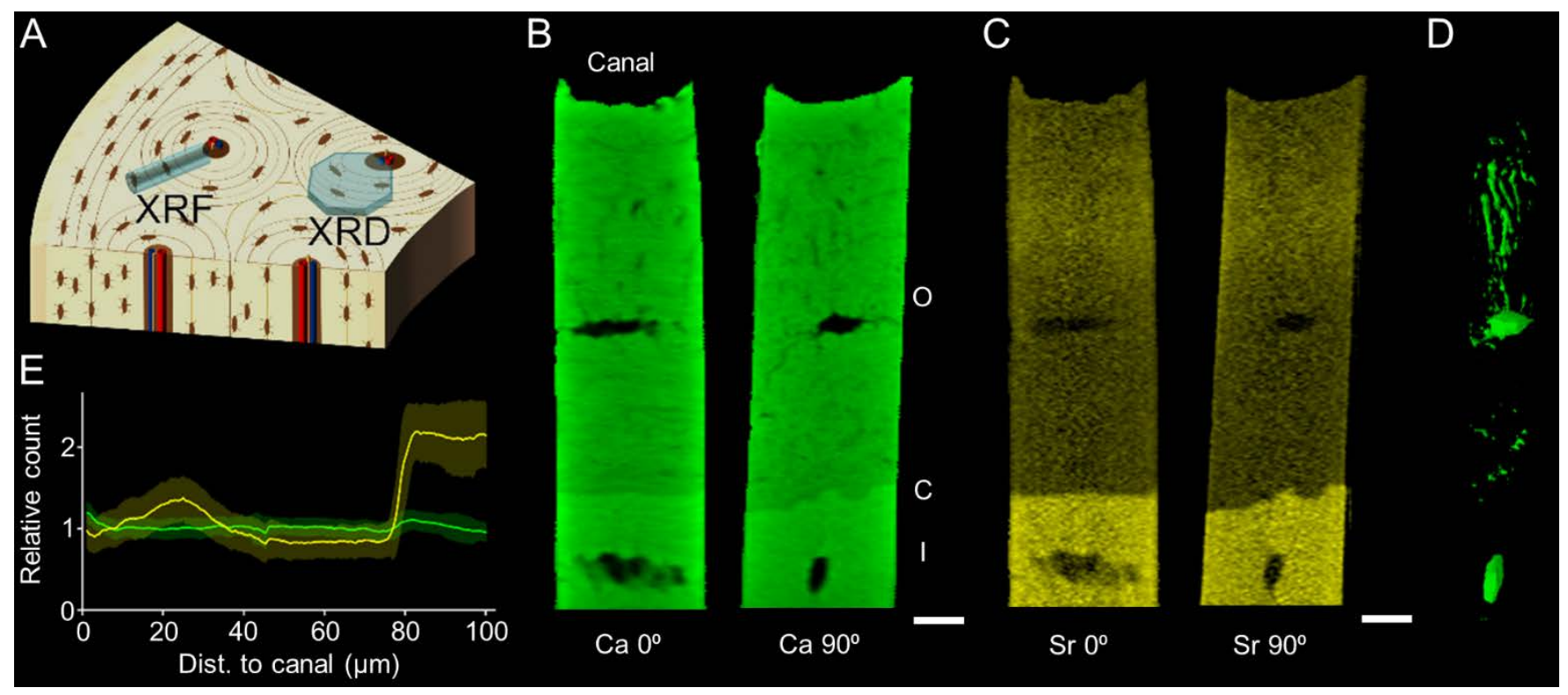

Figure 1. (A) Sketch of osteonal bone showing how the samples were oriented, (B) and (C) Reconstructed Ca (B) and Sr (C) fluorescence intensities in two orthogonal slices through the $3 \mathrm{D}$ volume. $\mathrm{O}$ indicates osteonal bone, $\mathrm{C}$ indicates position of cement line, and $\mathrm{I}$ indicates interstitial bone. (D) 3D rendering of Ca void space showing lacunae and canalicular extrusions, (E) Ca (green) and Sr (yellow) intensity as a function of distance from the osteon canal relative to mean fluorescence intensity inside the osteon. All scale bars are $10 \mu \mathrm{m}$. 
is not possible to distinguish peak broadening arising from size or micro-strain effects along the $\mathbf{a}$ and $\mathbf{b}$ directions in powder diffraction, thus imposing the assumption of needle shaped crystals; the broadening determined in the $a, b$-plane is therefore a weighted average of the broadening along these axes and it is not possible to establish whether crystals are in fact needle or platelet shaped. The average atomic displacement parameter, $U_{\text {iso }}$, describes a combination of several effects of 'disorder', both static and temperature dependent dynamic ones. ${ }^{56,57} \mathrm{We}$ used a single overall parameter to describe the combined average effect on all atoms. In the present study, we expect that contributions from thermal vibrations are to first order the same throughout the bone sample, whereas the level and type of defects and substitutions as well as the mineral interaction with the organic matrix might vary and are therefore most likely to be the main contributors to spatial variations in $U_{\text {iso }}$.

The distribution of the scale factor, i.e. the amount of crystalline material, in a single layer in the middle of the measured volume is shown in Figure 2A with a 3D rendering of the whole measured volume in Figure 2B. Weak pseudo-periodic variations radiating from the osteon central canal as well as an elevated scale factor close to the canal show that the degree of mineralization varies with distance from the blood vessel. The $z$ averaged scale factor, i.e. the average of the scale factor along the osteon long axis (Figure 2C), retains these features showing that they are preserved from layer to layer over the $10.4 \mu \mathrm{m}$ high volume. The variation between layers (Figure 2D) shows similar features to the $z$-averaged scale factor because of minute drifts in the position of lamellae along $z$. Additionally, increased variation along sample, lacunar and canalicular edges are observed due to their localized nature. This interlayer variation can stem from (1) the precision of the data, (2) edge effects, e.g. around osteocyte lacunae, and (3) biological variation between layers. The variation between layers (the average of the standard deviation along $z$ ) is compared to the in-layer variation (the average of the standard deviations of each layer) and the overall variation (the standard deviation of the entire measured

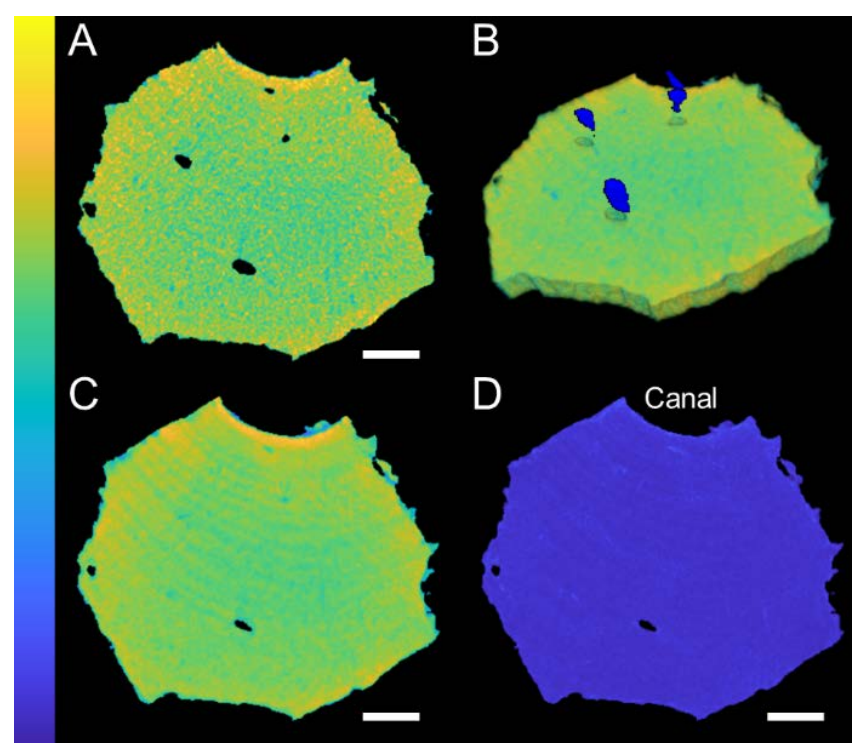

Figure 2. Amount of crystalline material. (A) Scale factor in a middle layer of the measured volume, (B) 3D rendering of scale factor with 3D rendering of void space superimposed, (C) z-averaged scale factor, (D) Standard deviation of scale factor between layers. Color bar $=0-3.5 \times 10^{-5}$ a.u. All scale bars are $20 \mu \mathrm{m}$ volume) in Table S1. The between-layer variation is smaller than both the total variation and the in-layer variation showing that the scale factor is pseudo-uniform along the osteon axis. Other crystallographic properties such as atomic displacement parameter ( $\left.U_{\text {iso }}\right)$ and diffraction peak widths also display lower between-layer variation (Figure S3B-G) compared to both the total and in-layer variation (Table S1). This continuity in the nanocrystal properties along the osteon axis demonstrates that the mineralization conditions are relatively constant along the forming osteon long axis over a distance of at least $10.4 \mu \mathrm{m}$ at all stages of bone growth. This height corresponds roughly to the diameter of an osteoblast. ${ }^{15}$

Figure 3A-G show z-averaged values of the crystallographic parameters along with their dependence on distance to the osteon central canal. Banding and/or pseudo-periodic variation radiating from the central canal is observed in several of the parameters, most prominently the scale factor (Figure $3 \mathrm{~A}$ ) and $U_{\text {iso }}$ (Figure 3B) that have similar periods of $\sim 5 \mu \mathrm{m}$. Both the amount of crystalline material and the local disorder in the crystals thus vary with a period that matches the reported lamellar thickness of 3-7 $\mu \mathrm{m}$ in osteons. ${ }^{19,58}$ The scale factor and $U_{\text {iso }}$ correlate positively (Figure $3 \mathrm{H}$ ) with a Pearson correlation coefficient of 0.60 showing that the crystals are more disordered in places with a high degree of mineralization. The observed periodic variation in the amount of crystalline material (scale factor) is in agreement with the work by Gupta et al. showing lamellar level modulation of both mineral content and mechanical properties. ${ }^{20}$ The observed banding of the scale factor and $U_{\text {iso }}$ is not concentric about the osteon central canal, which explains why the periodicity is smeared out for distances larger than $\sim 40 \mu \mathrm{m}$. Consequently, the bone remodeling event resulting in the investigated osteon must either have been initiated by osteoclasts drilling out a non-circular channel, or the rate of bone deposition by osteoblasts was not preserved along the edge of the osteon. Several structural parameters, e.g. the scale factor and $U_{\text {iso }}$ exhibit some banding in the standard deviation between measured layers (Figure S3A and B, respectively), because of small drifts in the position of lamellae along the osteon axis or small local variations in the material laid down at the same time.

The bone close to the osteon central canal contains crystals that are fundamentally different from the crystals in the remainder of the osteon as evidenced by all the extracted crystal parameters. To guide the eye, a white dashed line in the distance plots (Figure 3 ) marks a distance of $12.3 \mu \mathrm{m}$ from the canal. High scale factor values close to the osteon central canal match the increased $\mathrm{Ca}$ levels in the region of the sample investigated by XRF-CT suggesting that the canal-associated region in osteons is generally more highly mineralized compared to non-canal-associated osteonal bone. $U_{\text {iso }}$ also shows a distinct band of lower values near the osteon central canal corresponding to a lower degree of crystal disorder.

Figure $3 \mathrm{C}$ and D show unit cell $c / a$ and unit cell volume, respectively. Both values are lower in the region near the central canal, implicating a shortened unit cell $c$-axis. Differences in unit cell volumes are usually associated with differences in chemical composition in solid solutions like the heavily substituted bioapatite. The fact that bands of constant unit cell volumes are observed, especially close to the canal, suggests that the composition of the mineral was constant during the formation of each band. The mineral in the bone close to the osteon central canal is thus differently substituted compared to mineral located further away from the canal. 
Differences in diffraction peak widths are associated with effects of both crystallite size and micro-strain fluctuations. The broader the peak, the smaller and/or more strained the crystallites. ${ }^{55}$ Here, we do not separate the two effects, but report the FWHM $(\Gamma)$ of diffraction peaks broadened by size/strain along the $a(b)$ and $c$ axes in Figure $3 \mathrm{E}$ and F, respectively. Peak broadening from both directions increases close to the osteon central canal reflecting that the crystallites in this region are smaller and/or more strained compared to the crystallites located further away from the canal. This could indicate that the more recently formed crystals near the canal are still growing, but could also be a result of altered matrix composition in this region of bone (see below) that may affect how the crystallites are strained. ${ }^{53,54}$ Both $\Gamma_{\mathrm{a}, \mathrm{b}}$ and $\Gamma_{\mathrm{c}}$ display distinct banding around the canal, but are not spatially correlated. However, there is a spatial correlation between the bands with high values of $\Gamma_{\mathrm{a}, \mathrm{b}}$ or $\Gamma_{\mathrm{c}}$ and the regions of high inter-layer variation along
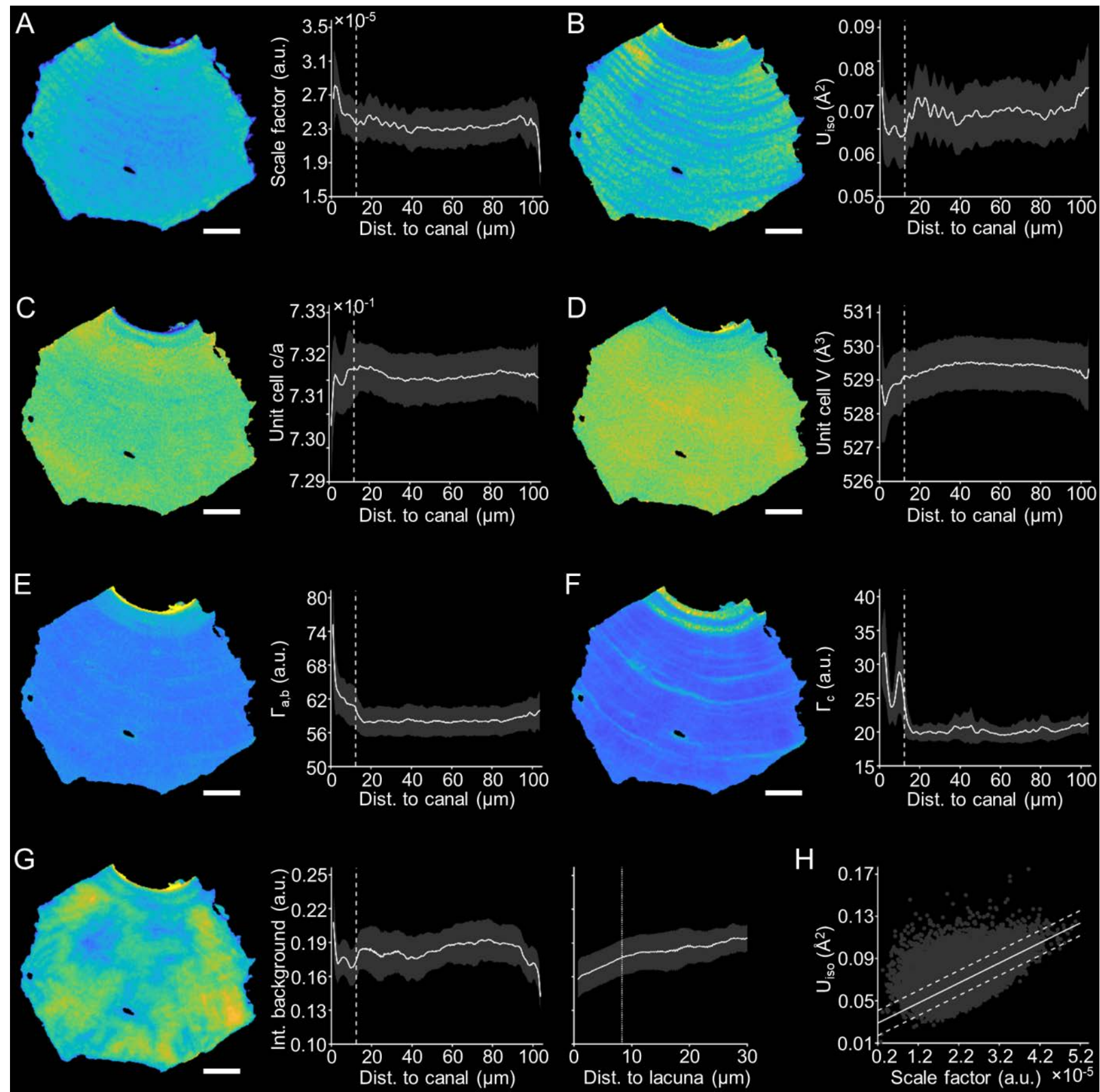

Figure 3. Maps of $z$-averaged crystallographic parameters and their development as a function of distance to the osteon canal (white dashed line marking $12.3 \mu \mathrm{m}$ ). (A) Scale factor, (B) $U_{\text {iso, }}(\mathrm{C})$ Unit cell $c / a$, (D) Unit cell $V$, (E) peak FWHM along the $a(b)$ axis $\left(\Gamma_{\mathrm{a}, \mathrm{b}}\right)$, (F) peak FWHM along the $c$ axis $\left(\Gamma_{\mathrm{c}}\right),(\mathrm{G})$ Integrated background signal (Int. Background) including dependence on distance to the nearest lacunar surface with the white dotted line marking the approximate transition between osteocyte associated bone matrix and "normal“ bone matrix, (H) Correlation between $U_{\text {iso }}$ and scale factor: data points (gray dots), linear fit with Pearson correlation coefficient of $\rho=0.60$ (solid white line) and $95 \%$ prediction intervals (dotted white lines). Color bar is the same as in Figure 2 with the range for the individual parameter matching the $y$-axes in the distance plots. All scale bars are $20 \mu \mathrm{m}$ 
the osteon axis (up to $15 \%$ and $25 \%$ in $\Gamma_{\mathrm{a}, \mathrm{b}}$ and $\Gamma_{\mathrm{c}}$, respectively) (Figure S3E and F). This speaks to a local variation in the bone material when layers of bone are laid down in the forming osteon. Note that the model assumes that the crystals are needle shaped, as it is not possible to distinguish differences along the $a$ and $b$ axes due to the hexagonal symmetry of apatite. Any differences in the real $a$ and $b$ axes will thus be averaged. Much debate exist on this matter, some studies indicating needle shaped crystals ${ }^{59,60,61}$ and others plate shaped crystals, ${ }^{62,63}$ with recent results suggesting that the mineral particles are plate shaped aggregates of needle shaped crystals. ${ }^{1}$ We stress that the present data cannot distinguish between the possible morphologies due to the hexagonal symmetry of apatite. Nevertheless the conclusions made above remain valid irrespective of the actual crystallite shape.

The integrated scattering background of the diffraction data can be used as a proxy for the distribution of organic or amorphous matter (Figure 3G). A clear band is seen in the canalassociated part of the osteon. In addition, volumes of lower integrated background signal surround the osteocyte lacunae with the integrated background increasing gradually with distance from the lacunar surface (Figure 3G). This signifies that the bone matrix closely associated with the cellular network is different compared to "normal" bone matrix. ${ }^{11,64}$ By linear fitting of the two regions observed in the plot, we find that this effect extends approximately $8.3 \mu \mathrm{m}$ from the lacunar surface indicating that osteocyte associated bone matrix makes up a significant fraction of the total bone volume. Bach-Gansmo et al. determined mean lacunar volumes and densities in human iliac crest cortical bone in men of $549 \mu^{3}$ and $15.2 \times 10^{3} \mathrm{~mm}^{-3}$, respectively, ${ }^{65}$ which corresponds to less than $1 \%$ lacunar porosity of the bone volume $(\mathrm{V} / \mathrm{V})$. However, the fraction of bone matrix affected by the osteocytes is large: approximating the lacunae as spheres and adding the $8.3 \mu \mathrm{m}$ lacuna-associated bone to the lacunar radius indicates that $15 \%$ of the bone volume is affected by osteocyte lacunae. Furthermore, shadows reminiscent of canaliculi can be seen in the inter-layer variation of the background and scale factor along the osteon axis (Figure S3G) because the canaliculi, a few hundred nanometers in diameter, will only intersect a few layers in the same in-plane position of the measured volume. They will give rise to lower diffraction/background signals compared to mineralized bone material and thus an increased inter-layer variation in the scale factor and background, respectively.

To investigate the generality of the above observations, crystallographic information of additional osteons was collected by 2D scanning of a large area of a $\sim 25 \mu \mathrm{m}$ thick bone slice. The results of Rietveld analysis on this 2D section are shown in Figure S4 and discussed in the supporting information.

The present findings of varying biomineral characteristics in osteonal bone suggest local variations in the biomineralization process. Several factors are known to influence and control the mineralization of bone such as non-collagenous proteins, ${ }^{66,67}$ polysaccharides, and citrate. ${ }^{68}$ In situ and ex situ characterization of apatite crystallization has shown that nano-crystalline apatite can form from a citrate-amorphous calcium phosphate precursor during desorption of citrate, ${ }^{47,}{ }^{69}, 70$ while solid-state NMR studies revealed that mineral platelet particles in bone are separated by citrate bridges ${ }^{71,72}$ and/or polysaccharides. ${ }^{68,73}$ The presence of a zone around the osteon central canal with different biomineral crystallographic properties may be related to the increased presence of non-collagenous protein fragments that are rich in the ASARM motif, e.g. osteopontin. These proteins are inhibitors of apatite formation in vitro while their roles in vivo remain incompletely understood. ${ }^{74}$ Their presence may stabilize metastable states during crystallization. ${ }^{75}$ Knockout of osteopontin results in increased degrees of mineralization and crystallinity but not to macroscopic skeletal deformities. ${ }^{76}$ Osteopontin is localized at mineralization foci during early stages of biomineralization ${ }^{77}$ and breakdown of osteopontin and similar proteins is an important step in the mineralization of especially perilacunar, pericanalicular and peri-canal bone. This process is regulated by PHEX and defects therein results in crosslinked hypophosphatemia. ${ }^{64,78}$ When PHEX is inactive, unmineralized halos appear, indicating that breakdown of osteopontin, and thus mineralization inhibiting properties, is an essential step in biomineralization of these compartments. The lower diffraction background signal around osteocyte lacunae observed herein reflects that the composition of the non-crystalline bone matrix is indeed different in the osteocyte-associated bone. The significant changes in mineral microstructure with smaller crystallite sizes around the blood vessel, is consistent with crystallite growth inhibition by ASARM (e.g. osteopontin) protein fragments of the forming crystals. Crystallite growth inhibition would result in smaller crystals while the total amount of crystalline material will be dependent on multiple factors including the overall Ca-influx during the crystallization process. This reflects that bone biomineralization is locally controlled by the expression of proteins and is the first testament of this local control on the formed biomineral.

\section{CONCLUSIONS}

In conclusion, we used XRF-CT and DSCT to reveal spatio/temporal variations in bone biomineralization. During Haversian remodeling a tunnel is carved out by osteoclasts, and osteoblasts subsequently fill in the tunnel with layers of bone ${ }^{18 \text {, }}$ ${ }^{19}$ resulting in a lamellar motif. As the bone is then mineralized, it is commonly believed that the crystallographic $c$-axis will conform to the orientation of the fibrils, ${ }^{79}$ but that the biomineral is otherwise the same throughout the bone. Here, we have shown, however, that the nature of the mineral varies across the osteon in terms of crystal composition, disorder, and crystallite size and/or microstrain. Surprisingly, this variation is not stochastic but occurs in bands corresponding to layers of bone laid down at the same point in time. Hence, it is likely that the variation observed is a reflection of the microenvironment during bone mineralization and that the processing of mineralization controlling proteins are reflected in the local biomineral properties. Bone biomineral thus varies in crystallographic and chemical properties dependent on the local environment during bone remodeling.

\section{METHODS}

Sample preparation. Samples of osteonal bone were taken near the mid-diaphysis of a human femur from a young male donor. To ensure that fluorescence signals were not affected by sample self-absorption and that diffraction signals were sufficiently intense, two samples were made (Figure 1A): One bone rod was prepared for DSCT (the XRD sample) and one for fluorescence tomography (the XRF sample). Both rods were made from bone cross sections cut perpendicular to the bone long axis and polished to a thickness of $\sim 80 \mu \mathrm{m}$ for the XRD sample and $\sim 30 \mu \mathrm{m}$ for the XRF sample by dry manual polishing using fine grain $(5 \mu \mathrm{m})$ polishing paper. The XRD rod was cut with a standard razor blade from a suitable osteon to obtain an $80 \mu \mathrm{m}$ 
high octagon with one of the sides coinciding with the osteon central canal and a diameter of $\sim 130 \mu \mathrm{m}$ corresponding approximately to the osteon radius (Figure 1A). The XRF sample was prepared from another suitable osteon by a combination of razor blade cutting and focused ion beam milling (Versa 3D, FEI, Eindhoven, the Netherlands). This resulted in an octagonal rod with a diagonal of $\sim 30 \mu \mathrm{m}$ and a length of $\sim 120 \mu \mathrm{m}$ starting from the osteon central canal (Figure 1A). In addition, a thin bone section was prepared for 2D scans with a thickness of $\sim 25$ $\mu \mathrm{m}$.

Data collection. Transmission X-ray diffraction and X-ray fluorescence datasets were collected at the hard X-ray micro probe endstation of the P06 beamline at PETRA-III, DESY, Hamburg, Germany. ${ }^{80,81}$ The X-ray energy was selected as 16.5 $\mathrm{keV}$ and Kirkpatrick-Baez mirrors were used to focus the beam to $400 \times 400 \mathrm{~nm}^{2}$. The fluorescence signal was recorded with a Maia 384-C X-ray fluorescence detector placed $\sim 1 \mathrm{~mm}$ upstream of the sample with an exposure time of $15 \mathrm{~ms} .{ }^{82}$ The diffraction signal was recorded in the $q$-range from $4.7-52.0 \mathrm{~nm}^{-}$ ${ }^{1}$ with an Eiger 4M detector (Dectris, Baden-Daettwill, Switzerland) placed $13.3 \mathrm{~cm}$ downstream from the sample and with an exposure time of $50 \mathrm{~ms}$. The sample-to-detector distance and detector tilt of the diffraction detector were calibrated with a $\mathrm{LaB}_{6}$ standard sample. 2D projections were built by translation of the samples through the beam in steps of $400 \mathrm{~nm}$ to cover an area of $46 \mu \mathrm{m} \times 126 \mu \mathrm{m}$ (horizontal $\times$ vertical) for the XRF sample and an area of $150 \mu \mathrm{m} \times 10.4 \mu \mathrm{m}$ (horizontal $\times$ vertical) for the XRD sample including air on both sides of the samples. Full tomographic datasets were assembled by repeating the translational pattern for a number of viewing angles in a sequence determined by the golden angle method. ${ }^{83}$ For the XRF sample, 104 projections were measured resulting in a total of $>3.8$ million fluorescence spectra, and for the XRD sample, 270 projections were measured resulting in a total of $>2.6$ million diffraction patterns. Additionally, 2D XRD maps covering several osteons were measured on a thin bone slice mounted with the bone long axis parallel to the beam.

Reconstruction. The fluorescence spectra were fitted with the GeoPIXE-software package (CSIRO, c/o CMSE, Clayton VIC, Australia) ${ }^{84,85}$ followed by individual reconstruction of the fitted intensity of each element of interest using filtered back projection with a Hann filter. Prior to reconstruction, the 2D detector images of the diffraction rings were convoluted with a $2 \mathrm{D}$ Gaussian filter to reduce detector discretization induced noise and azimuthally integrated into 1D diffractograms using in house Matlab scripts (R2017b, MathWorks, Natick, MA). A reconstruction was performed in each $2 \theta$ bin using filtered back projection with a Hann filter to obtain a full diffractogram in every volume element from within the sample ( $>3.6$ million voxels).

Rietveld refinements. The resulting position resolved diffractograms were Rietveld refined individually in the software MultiRef, ${ }^{47,48,49}$ which is a Matlab interface to GSAS ${ }^{86}$ that allows setting up automatic refinements in an intelligent way. MultiRef runs several refinement passes across vast datasets with the model being updated automatically according to user settings between each refinement pass based on the results of the previous run. The same starting model was used in all points based on manual refinement of a representative data point starting from a HAP model. The refinements were set up in five passes, each one adding more complexity to the model. In the first pass, only the scale factor and the background coefficients were re- fined using a six coefficients Chebyshev polynomial description of the background. For the second pass, only voxels likely to contain HAP, as evaluated by the level of the scale factor compared to the estimated standard deviation of the scale factor, were included. In this pass, preferred orientation described by a $10^{\text {th }}$ order spherical harmonics function was added to the model and refined. In the third pass, the lattice parameters were allowed to vary. Voxels containing HAP, as evaluated by the size of the scale factor, were refined in a $4^{\text {th }}$ cycle where refinement of profile parameters were added to the model and the refinement stabilized by Marquardt dampening. The peak profiles were modelled by a pseudo-Voigt profile function including a Lorentzian strain broadening parameter and two parameters describing the anisotropic crystal shape of HAP in bone that was taken to be needle shaped with the $c$-axis being the anisotropic axis. Atomic displacement parameters $\left(U_{\text {iso }}\right)$ were added in the last refinements pass with the constraint that their magnitude should be equal for all atoms.

Data analysis. All results were evaluated as a function of distance to the osteon central canal by calculating the distance transform to the canal surface. This was done in $3 \mathrm{D}$ for the reconstructed fluorescence signals, while the distance transform was calculated for each layer separately for the XRD sample. The canal surface outside of the XRD sample was estimated in each layer with a circle fitted to the measured part of the surface. The distance binning was kept equal to the voxel size, i.e. 400 $\mathrm{nm}$. The crystallographic parameters were additionally evaluated as a function of the distance to the nearest lacuna surface. Correlations between crystallographic parameters were checked to test that the level of freedom in the applied Rietveld model was appropriate.

\section{ASSOCIATED CONTENT}

\section{Supporting Information}

The supporting information is available free of charge via the Internet at http://pubs.acs.org.

Figures S1-S5, Table S1 and detailed description of analysis of bone area characterized by 2D scanning XRD.

\section{AUTHOR INFORMATION}

\section{Corresponding Author}

*hbirkedal@chem.au.dk.

\section{Author Contributions}

All authors have given approval to the final version of the manuscript

\section{ACKNOWLEDGMENT}

We acknowledge DESY (Hamburg, Germany), a member of the Helmholtz Association HGF, for the provision of experimental facilities. Parts of this research were carried out at PETRA III and we thank the beamline staff for assistance in using the hard x-ray microprobe endstation of the P06 beamline. In addition, we thank EU CALIPSOplus Transnational Access program for financial travel support. We thank the Danish Agency for Science, Technology, and Innovation for funding (DANSCATT). We thank the Villum Foundation (grant 17553) and Independent Research Fund Denmark | Natural Sciences (grant 8021-00237B) for funding. Affiliation with the center for integrated materials research (iMAT) at Aarhus University is gratefully acknowledged. 


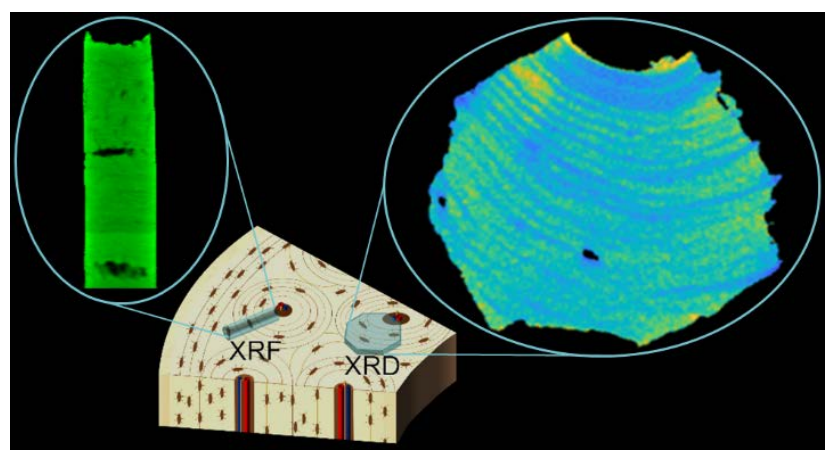

\section{REFERENCES}

1. Reznikov, N.; Bilton, M.; Lari, L.; Stevens, M. M.; Kröger, R. Fractal-Like Hierarchical Organization of Bone Begins at the NanoscaleScience2018 360, eaao2189.

2. Stock, S. R. The Mineratollagen Interface in BoneCalcif. Tissue Int2015 97, 262-280.

3. Zimmermann, E. A.; Ritchie, R. O. Bone as a Structural MateriaAdv. Healthcare Mater. 2015 4, 12871304.

4. Launey, M. E.; Buehler, M. J.; Ritchie, R. $\mathrm{O}$. On the Mechanistic Origins of Toughness in Bone.Annu. Rev. Mater. Reß010 40, 2553. 5. Currey, J. D.,Bones : Structure and Mechanics Second edition. ed.; Princeton University Press: Princeton, N.J. ; Woodstock, 2002.

6. Barthelat, F.; Yin, Z.; Buehler, M. J. Structure and Mechanics of Interfaces in Biological Materials.Nat. Rev. Mate/2016, 1, 16007.

7. Wegst, U. G. K.; Bai, H.; Saiz, E.; Tomsia, A. P.; Ritchie, R. O. Bioinspired Structural MaterialsßNat. Mater.2015 14, 2336. 8. Meyers, M. A.; McKittrick, J.; Chen, P. Y. Structural Biological Materials: Critical MechanicsMaterials Conections.Science2013 339, 773779.

9. Dunlop, J. W. C.; Fratzl, P. Biological CompositesAnnu. Rev. Mater. Rez010 40, 1 24.

10. Reznikov, N.; Steele, J. A. M.; Fratzl, P.; Stevens, M. M. A Materials Science Vision of
Extracellular Matrix Mineraliztion. Nat. Rev. Mater.2016 1, 16041.

11. Reznikov, N.; Shahar, R.; Weiner, S. Bone Hierarchical Structure in Three Dimensions Acta Biomater2014, 10, 3815 3826.

12. Betts, F.; Blumenthal, N. C.; Posner, A. S. Bone MineralizationJ. Cryst. Growth981, 53, 6373.

13. Wopenka, B.; Pasteris, J. D. A Mineralogical Perspective on the Apatite in Bone.Mater. Sci. Eng., 2005 25, 131143. 14. Havers, C.,Osteologia Nova, or, Some New Observations of the Bones, and the Parts Belonging to Them, with thdanner of Their Accretion and NutritionSamual Smith: London, 1691.

15. Lassen, N. E.; Andersen, T. L.; Ploen, G. G.; Soe, K.; Hauge, E. M.; Harving, S.; Eschen, G. E. T.; Delaisse, J. M. Coupling of Bone Resorption and Formation in Real Time: New Knowledge Gained from Human Haversian BMUs. J. Bone Miner. Re\&017, 32, 13951405. 16. BachGansmo, F. L.; Weaver, J. C.; Jensen, M. H.; Leemreize, H.; Mader, K. S.; Stampanoni, M.; Brüel, A.; Thomsen, J. S.; Birkedal, H. Osteocyte Lacunar Properties in Rat Cortical Bone: Differences between Lamellar and Central BoneJ. Struct. Bio/2015 191, 5967. 17. BachGansmo, F. L.; Irvine, S. C.; Bruel, A.; Thomsen, J. S.; Birkedal, H. Calcified Cartilage Islands in Rat Cortical Bonealcif. Tissue Int2013 92, 330338.

18. GiraudGuille, M. M. Twisted Plywood Architecture of Collagen Fibrils in Human Compact Bone OsteonEalcif. Tissue Int1988 42, 167180. 
19. Wagermaier, W.; Gupta, H. S.; Gourrier, A.; Burghammer, M.; Roschger, P.; Fratzl, P. Spiral Twisting of Fiber Orientation inside Bone Lamellae.Biointerphase8006 1, 15.

20. $\quad$ Gupta, H. S.; Stachewicz, U.; Wagermaier, W.; Roschger, P.; Wagner, H. D.; Fratzl, P. Mechanical Modulation at the Lamellar Level in Osteonal BoneJ. Mater. Res2006 21, 19131921.

21. Wittig, N. K.; Laugesen, M.; Birkbak, M. E.; BachGansmo, F. L.; Pacureanu, A.; Wendelboe, M. H.; Bruns, S.; Sørensen, H. O.; Brüel, A.; Thomsen, J. S.; Birkedal, H.

Canalicular Junctions in the Osteocyte Lacuno Canalicular Network of Cortical Bonel.cs Nano 2019 13, 64216430.

22. Bonewald, L. F. The Amazing Osteocyte. J. Bone Miner. Re\&011, 26, 229238.

23. Ebacher, V.; Wang, R. Z. A Unique Microcracking Process Associated with the Inelastic Deformation of Haversian Bonfdv. Funct. Mater.2009 19, 5766.

24. O'Brien, F. J.; Taylor, D.; Lee, T. C. Bone as a Composite Material: The Role of Osteons as Barriers to Crack Growth in Compact Borket. J. Fatigue2007, 29, 10511056.

25. Seto, J.; Gupta, H. S.; Zaslansky, P.; Wagner, H. D.; Fratz $\mathbb{P}$. Tough Lessons from Bone: Extreme Mechanical Anisotropy at the MesoscaleAdv. Funct. Mater2008 18, 1905 1911.

26. Koester, K. J.; Ager, J. W.; Ritchie, R. O. The True Toughness of Human Cortical Bone Measured with Realistically Short Crack\&at.

Mater. 2008 7, 672677.

27. Peterlik, H.; Roschger, P.; Klaushofer, K.; Fratzl, P. From Brittle to Ductile Fracture of Bone.Nat. Mater.2006 5, 5255.

28. Hert, J.; Fiala, P.; Petrtyl, M. Osteon Orientation of the Diaphysis of the Long Bones in Man. Bone1994 15, 269277.

29. de Jonge, M. D.; Holzner, C.; Baines, S. B.; Twining, B. S.; Ignatyev, K.; Diaz, J.; Howards, D. L.; Legnini, D.; Miceli, A.; McNulty, I.; Jacobsen, C. J.; Vogt, S. Quantitative 3D Elemental Microtomography of Cyclotella Meneghiniamat 400nm Resolution. PNAS2010 107, 156715680.

30. de Jonge, M. D.; Vogt, S. Hard-Xay Fluorescence Tomographan Emerging Tool for Structural VisualizationCurr. Opin. Struct. Biol. 2010 20, 606614.

31. Birkbak, M. E.; Leemreize, H.; Frolich, S.; Stock, S. R.; Birkedal, H. Diffraction

Scattering Computed Tomography: A Window into the Structures of Complex Nanomaterials.

Nanoscale2015 7, 1840218410.

32. Leemreize, H.; Almer, J. D.; Stock, S. R.; Birkedal, $\mathrm{H}$. ThreeDimensional Distribution of Polymorphs and Magnesium in a Calcified Underwater Attachment System by Diffraction Tomography J. R. Soc., Interfacz013 10.

33. Alvarez-Murga, M.; Bleuet, P.; Hodeau, J. L. Diffraction/Scattering Computed

Tomography for Thre巴imensional Characterizatio of Multi-Phase Crystalline and Amorphous MaterialsJ. Appl. Crystallogr2012, 45, 11091124.

34. Beale, A. M.; Jacques, S. D. M.; Gibson, E. K.; Di Michiel, M. Progress Towards Five Dimensional Diffraction Imaging of Functional Materials under Proce $\$$ onditions.Coord.

Chem. Rev2014 277, 208223.

35. Bleuet, P.; Welcomme, E.; Dooryhee, E.; Susini, J.; Hodeau, J. L.; Walter, P. Probing the Structure of Heterogeneous Diluted Materials by Diffraction TomographyNat. Mater.2008 7, 468-472.

36. Stock,S. R.; Almer, J. D. Diffraction Microcomputed Tomography of an Alatrix SiC-Monofilament Compositel. Appl.

Crystallogr.2012, 45, 10771083.

37. Leemreize, H.; Birkbak, M.; Frolich, S.; Kenesei, P.; Almer, J. D.; Stock, S. R.; Birkedal, $H$. Diffraction Computed Tomography Reveals the Inner Structure of Complex Biominerals. Developments in-※ay Tomography I8014 9212, 92120C.

38. Stock, S. R.; de Carlo, F.; Almer, J. D. High Energy XRay Scattering Tomography Applied to Bone J. Struct. Biol2008 161, 144 150.

39. Golosio, B.; Simionovici, A.; Somogyi, A.; Lemelle, L.; Chukalina, M.; Brunetti, A. Internal Elemental Microanalysis Combining $X$ Ray Fluorescence, Compton and Transmission Tomography J. Appl. Phys2003 94, 145156.

40. Hesse, B.; Varga,.PLanger, M.;

Pacureanu, A.; Schrof, S.; Mannicke, N.; Suhonen, H.; Maurer, P.; Cloetens, P.; Peyrin, F.; Raum, K. Canalicular Network Morphology Is 
the Major Determinant of the Spatial Distribution of Mass Density in Human Bone Tissue:

Evidence by Means of Synchrotron Radiation Phase-Contrast Nano-CT. J. Bone Miner. Res. 2015 30, 346356.

41. You, L. D.; Weinbaum, S.; Cowin, S. C.; Schaffler, M. B. Ultrastructure of the Osteocyte Process and Its Pericellular Matrixnat. Rec. $A$. Discov. Mol. Cell. EvolBiol. 2004 278A, 505 513.

42. Akkus, O.; PolyakovæAkkus, A.; Adar, F.; Schaffler, M. B. Aging of Microstructural Compartments in Human Compact BodeBone Miner. Res2003 18, 10121019.

43. Pemmer, B.; Roschger, A.; Wastl, A.; Hofstaetter, J. G.; Wobrauschek, P.; Simon, R.; Thaler, H. W.; Roschger, P.; Klaushofer, K.; Streli, C. Spatial Distribution of the Trace Elements Zinc, Strontium and Lead in Human Bone TissueBone2013 57, 184193.

44. Li, C.; Paris, O.; Siegel, S.; Roschger, P.; Paschalis, E. P.; Klaushofer, K.; Fratzl, P. Strontium Is Incorporated into Mineral Crystals Only in Newly Formed Bone During Strontium Ranelate Treatment. Bone Miner. Re\&010 25, 968975.

45. Brüel, A; Olsen, J.; Birkedal, H.; Risager, M.; Andreassen, T. T.; Raffalt, A. C.; Andersen, J. E. T.; Thomsen, J. S. Strontium Is Incorporated into the Fracture Callus but Does Not Influence the Mechanical Strength of Healing Rat Fracture calcif. Tissue Int2011, 88, 142152.

46. Bunger, M. H.; Oxlund, H.; Hansen, T. K.; Sorensen, S.; Bibby, B. M.; Thomsen, J. S.; Langdahl, B. L.; Besenbacher, F.; Pedersen, J. S. Birkedal, H. Strontium and Bone Nanostructure in Normal and Ovariectomized Rats Investigated by Scanning SmałAngle X-Ray Scattering. Calcif. Tissue Int2010 86, 294306.

47. Frolich, S.; Birkedal, H. Multiref: Software Platform for Rietveld Refinement of Multiple Powder Diffractograms fromin Situ Scanning or Diffraction Tomography Experiments J. Appl. Crystallogr.2015 48, 20192025.

48. Frolich, S.; Leemreize, H.; Jakus, A.; Xiao, X.; Shah, R.; Birkedal, H.; Almer, J. D.; Stock, S. R. Diffraction Tomography and Rietveld Refinement of a Hydroxyapatite Bone
Phantom J. Appl. Crystallogr2016 49, 103 109.

49. Birkbak, M. E.; Nielsen, I. G.; Frolich, S.; Stock, S. R.; Kenesei, P.; Almer, J. D.; Birkedal, $\mathrm{H}$. Concurrent Determination of Nanocrystal Shape and Amorphous Phases in Complex Materials by Diffraction Scattering Computed Tomography J. Appl Crystallogr.2017, 50, 192 197.

50. Vamvakeros, A.; Jacques, S. D. M.; Di Michiel, M.; Matras, D.; Middelkoop, V.; Ismagilov, I. Z.; Matus, E. V.; Kuznetsov, V. V.; Drnec, J.; Senecal, P.; Beale, A. M. operando Tomographic Diffraction Imaging of a Catalyst Bed. Nat. CommurRo18 9.

51. Elliott, J. C., Structure and Chemistry of the Apatites and Other Calcium Orthophosphate£lsevier: Amsterdam ; London, 1994.

52. Hidouri, M.; Dorozhkin, S. V.; Albelaḍi N. Thermal Behavior, Sintering and Mechanical Characterization of Multiple Ioßsubstituted Hydroxyapatite Bioceramics]. Inorg. Organomet. Polym. Mate2019 29, 87100.

53. Ibsen, C. J. S.; Birkedal, H. Modification of BoneLike Apatite Nanoparticle iße and Growth Kinetics by Alizarin Red SVanoscale 2010 2, 24782486.

54. Pokroy, B.; Quintana, J. P.; Caspi, E. N.; Berner, A.; Zolotoyabko, E. Anisotropic Lattice Distortions in Biogenic AragoniteVat. Mater. 2004, 3, 900902.

55. Clearfield, A.; Rebenspies, J. H.; Bhuvanesh, N.Principles and Applications of Powder Diffraction Blackwell: Oxford, 2008. S.56. Dunitz, J. D.; Schomaker, V.; Trueblood, K. N. Interpretation of Atomic Displacement Parameters from Diffraction Studies of Crystals. J. Phys. Gem.1988 92, 856867.

57. Burgi, H. B.; Capelli, S. C. Dynamics of Molecules in Crystals from Multemperature Anisotropic Displacement Parameters. I. Theory. Acta Crystallogr., Sect. A: Found. Aø000 56, $403-412$.

58. Weiner, S.; Traub, W.; Wagneti, D. Lamellar Bone: StructufEunction RelationsJ. Struct. Biol.1999 126, 241255.

59. FernándełMorán, H.; Engström, A. Electron Microscopy and - Ray Diffraction of Bone.Biochim. Biophys. Act $\$ 957,23,260264$. 
60. $\quad$ Fratzl, P.; Groschner, M.; Vogl, G.; Plenk, H.; Eschberger, J.; Fratzlzelman, N.; Koller, K.; Klaushofer, K. Mineral Crystals in Calcified Tissues - a Comparative-Study by SAXS. J. Bone Miner. Re\$992 7, 329334.

61. Traub, W.; Arad, T.; Weiner, S. Origin of Mineral CrystałGrowth in Collagen Fibrils. Matrix 1992 12, 251255.

62. Wachtel, E.; Weiner, S. SmaAngle XRay-Scattering Study of Dispersed Crystals from Bone and Tendon.. Bone Miner. Re\$994 9, 1651-1655.

63. Weiner, S.; Price, P. A. Disaggregation of Bone into CrystalsCalcif. Tissue Int1986 39, 365-375.

64. Barros, N. M. T.; Hoac, B.; Neves, R. L.; Addison, W. N.; Assis, D. M.; Murshed, M.; Carmona, A. K.; McKee, M. D. Proteolytic Processing of Osteopontin by Phex and Accumulation of Osteopontin Fragments in Hyp Mouse Bone, the Murine Model of- Xinked Hypophosphatemial. Bone Miner. Re\&013 28, 688699.

65. BachGansmo, F. L.; Brüel, A.; Jensen, M. V.; Ebbesen, E. N.; Birkedal, H.; Thomsen, J. S. Osteocyte Lacunar Properties and Cortical Microstructure in Humalliac Crest as a Function of Age and Seßone2016 91, 1119. 66. George, A.; Veis, A. Phosphorylated Proteins and Control over Apatite Nucleation, Crystal Growth, and Inhibitionchem. Rev2008 108, 46704693.

67. Murshed, M.; McKee, M. D. Molecular Determinants of Extracellular Matrix Mineralization in Bone and Blood Vesseksurr. Opin. Nephrol. Hyperten̊010 19, 359365.

68. Nikel, O.; Laurencin, D.; Bonhomme, C.; Sroga, G. E.; Besdo, S.; Lorenz, A.; Vashishth, D. Solid State NMR Investigation dritact Human Bone Quality: Balancing Issues and Insight into the Structure at the Orgatilineral Interface.J. Phys. Chem. 2012, 116, 6320 6331.

69. Chatzipanagis, K.; Iafisco, M.; Ronєal Herrero, T.; Bilton, M.; Tampieri, A.; Kroger, R.; DelgadoLopez, J. M. Crystallization of Citrate Stabilized Amorphous Calcium Phosphate to Nanocrystalline Apatite: A Surfadlediated Transformation CrystEngComr2016 18, 3170 3173.
70. Jensen, A. C. S.; Ibsen, C. J. S.; Sutherland, D.; Birkedal, H. Transparent Aggregates of Nanocrystalline Hydroxyapatite. Cryst. Growth Des2014 14, 63436349.

71. Davies, E.; Muller, K. H.; Wong, W. C.; Pickard, C. J.; Reid, D. G.; Skepper, J. N.; Duer, M. J. Citrate Bridges between Mineral Platelets in Bone.Proc. Natl. Acad. ScL. S. A.2014, 111, E1354E1363.

72. Hu, Y. Y.; Rawal, A.; SchmidRohr, K. Strongly Bound Citrate Stabilizes the Apatite Nanocrystals in BoneProc. Natl. Acad. Sci. U.

S. A.2010 107, 2242522429.

73. Wise, E. R.; Maltsev, S.; Davies, M. E.; Duer, M. J.; Jaeger, C.; Loveridge, N.; Murray, R. C.; Reid, D. G. The Organidineral Interface in Bone Is Predominantly Polysaccharidedem. Mater. 2007, 19, 50555057.

74. Hunter, G. K. Role of Osteopontin in Modulation of Hydroxyapatite Formatioralcif. Tissue Int2013 93, 348354.

75. Ibsen, C. J. S.; Gebauer, D.; Birkedal, H. Osteopontin Strongly Stabilizes Metastable States Prior to Nucleation During Apatite Formation.Chem. Mater2016 28.

76. Boskey, A L.; Spevak, L.; Paschalis, E.; Doty, S. B.; McKee, M. D. Osteopontin Deficiency Increases Mineral Content and Mineral Crystallinity in Mouse BoneCalcif. Tissue Int2002 71, 145154.

77. McKee, M. D.; Nanci, A. Osteopontin: An Interfacial ExtracellulaMatrix Protein in Mineralized TissuesConnect. Tissue Re\$996 35, 197205.

78. Boukpessi, T.; Hoac, B.; Coyac, B. R.; Leger, T.; Garcia, C.; Wicart, P.; Whyte, M. P.; Glorieux, F. H.; Linglart, A.; Chaussain, C.; McKee, M. D. Osteopontin and the Dento Osseous Pathobiology of 凶nked HypophosphatemiaBone2017, 95, 151161. 79. Frazl, P.; Gupta, H. S.; Paschalis, E. P.; Roschger, P. Structure and Mechanical Quality of the CollagerMineral NanoComposite in Bone.

J. Mater. Chemro04 14, 21152123.

80. Schroer, C. G.; Boye, P.; Feldkamp, J. M.; Patommel, J.; Samberg, D.; Schropp, A. Schwab, A.; Stephan, S.; Falkenberg, G.; Wellenreuther, G.; Reimers, N. HardRkay Nanoprobe at Beamline P06 at Petra/Nucl. Instrum. Methods Phys. Res., Sec2010 616, 93-97. 
81. Boesenberg, U.; Ryan, C. G.; Kirkham, R.; Siddons, D. P.; Alfeld, M.; Garrevoet, J.;

Nunez, T.; Claussen, T.; Kracht, T.; Falkenberg, G. Fast X-Ray Microfluorescence Imaging with Submicrometer-Resolution Integrating a Maia Detector at Beamline P06 at PetraIII. J.

Synchrotron Radia2016 23, 15501560.

82. Siddons, D. P, Kirkham, R.; Ryan, C. G.; De Geronimo, G.; Dragone, A.; Kuczewski, A. J.; Li, Z. Y.; Carini, G. A.; Pinelli, D.; Beuttenmuller, R.; Elliott, D.; Pfeffer, M.; Tyson, T. A.; Moorhead, G. F.; Dunn, P. A. Maia Ray Microprobe Detector Array Systen2nd Irt. Congr. XRay Opt. Microanal2014 499.

83. Kohler, T. A Projection Access Scheme for Iterative Reconstruction Based on the Golden Section.2004 IEEE Nucl. Sci. Symp. Conf. Rec., Vols 172004 39613965.

84. Ryan, C. G.; Cousens, D. R.; Sie, S. H.; Griffin, W. L.; Suter, G. F.; Clayton, E.

Quantitative Pixe Microanalysis of Geological Material Using the Csiro Proton Microprobe. Nucl. Instrum. Methods Phys. Res., Sea9\$0, 47, 5571.

85. Ryan, C. G.; Kirkham, R.; Hough, R. M.; Moorhead, G.; SiddonQ. P.; de Jonge, M. D.; Paterson, D. J.; De Geronimo, G.; Howard, D. L.; Cleverley, J. S. Elemental- Xay Imaging Using the Maia Detector Array: The Benefits and Challenges of Large Soliângle. Nucl. Instrum. Methods Phys. Res., Secro1la 619, 3743. 86. Larson, A. C.; Dreele, R. B. General Structure Analysis System (GSA\&)os Alamos Natl. Lab., [Rep.] LA (U. S2004 